\title{
WALKING AROUND THE BRAUER TREE
}

\author{
Dedicated to the memory of Hanna Neumann
}

\author{
J. A. GREEN
}

(Received 22 September 1972)

Communicated by M. F. Newman

\section{Introduction}

Let $G$ be a finite group, and $k$ a field of finite characteristic $p$, such that the polynomial $x^{|G|}-1$ splits completely in $k[x]$. Let B be a $k G$-block which has defect group $D$ which is cyclic of order $p^{d}(d \geqq 1)$. Brauer showed in a famous paper [2] that, in case $d=1$, the decomposition matrix of $\mathbf{B}$ is determined by a certain positive integer $e$ which divides $p-1$, and a tree $\Gamma$, a connected acyclic linear graph of $e+1$ vertices and $e$ edges. Twenty-five years later Dade ([3]) extended Brauer's theorem to the general case.

Dade shows that B contains $v+\left(p^{d}-1\right) / e$ simple $(=$ irreducible $)$ ordinary characters $X_{1}, \cdots, X_{e}$ and $X_{\lambda}(\lambda \in \Lambda)$, where $\Lambda$ is an index set containing $\left(p^{d}-1\right) / e$ elements. $B$ has $e$ simple modular characters $\phi_{0}, \cdots, \phi_{e-1}$; denote by $\eta_{0}, \cdots, \eta_{e-1}$ the corresponding projective indecomposable characters.

Put $X_{e+1}=\Sigma_{\lambda \in \Lambda} X_{\lambda}$. For each $i \in\{0, \cdots, e-1\}$ there is an equation

$$
\eta_{i}=X_{i(1)}+X_{i(2)}
$$

where $i(1), i(2) \in\{1, \cdots, e+1\}, i(1) \neq i(2)$ (see [3, section 7]). The Brauer tree $\Gamma$ of $\mathbf{B}$ is defined to have $\Gamma_{v}=\left\{X_{1}, \cdots, X_{e+1}\right\}$ as set of vertices, $\Gamma_{e}=\left\{\eta_{0}, \cdots, \eta_{e-1}\right\}$ as set of edges, and $\eta_{i} \in \Gamma_{e}$ is incident with $X_{j} \in \Gamma_{v}$ if and only if $X_{j} \in\left\{X_{i(1)}, X_{i(2)}\right\}$.

Let $R$ be a complete discrete valuation ring of characteristic zero, which has $k$ as residue-class field. An $R G$-lattice $A$ is an $R G$-module which is free and finitely-generated as $R$-module; $A$ affords a character, which we regard as ordinary character of $G$. Let $I=\{0, \cdots, e-1\}$, and let $W_{i}(i \in I)$ be a full set of projective indecomposable $R G$-modules; these are $R G$-lattices, and we arrange them so that $W_{i}$ has character $\eta_{i}$, for all $i \in I$.

The main purpose of this paper is to prove 
Theorem 2. Let $G, \mathrm{~B}, D, \Gamma$ be as above. Then

(i) The numbering of $\eta_{0}, \cdots, \eta_{e-1}$ can be so chosen, that there exists a family $\left(A_{n}\right)_{n \in Z}$ of RG-lattices, and a permutation $\delta$ of the set $I=\{0, \cdots, e-1\}$, such that there exist $R G$-short exact sequences

$$
\begin{aligned}
& \mathrm{E}_{2 i}: 0 \rightarrow A_{2 i+1} \rightarrow W_{\delta(i)} \rightarrow A_{2 i} \rightarrow 0 \\
& \mathrm{E}_{2 i+1}: 0 \rightarrow A_{2 i+2} \rightarrow W_{i+1} \rightarrow A_{2 i+1} \rightarrow 0,
\end{aligned}
$$

for all $i \in Z$. Here $i$ is to be taken $\bmod e$, so that $W_{n} \cong W_{n+e}$ and $A_{n} \cong A_{n+2 e}$, for all $n \in Z$.

(ii) The $2 e R G$-modules $A_{0}, \cdots, A_{2 e-1}$ are mutually non-isomorphic, so that the sequences $\mathrm{E}_{n}(n \geqq 0)$ provide a projective $R G$-resolution of $A_{0}$ which is periodic of period $2 e$ :

$$
\cdots \rightarrow W_{0} \rightarrow W_{\delta(e-1)} \rightarrow W_{e-1} \rightarrow \cdots \rightarrow W_{\delta(1)} \rightarrow W_{1} \rightarrow W_{\delta(0)} \rightarrow A_{0} \rightarrow 0 .
$$

In case $\mathrm{B}=\mathrm{B}_{0}(G)$ is the principal block of $G$, we can take $A_{0}=R_{G}$, which is regarded as trivial $R G$-module.

(iii) The character $P_{n}$ of $A_{n}$ belongs to $\Gamma_{v}$, for all $n \in Z$.

(iv) Suppose that B is a self-dual block, so that $\phi_{1} \in \mathrm{B}$, for all $i \in I$. Define permutations $\beta, \gamma$ of $I$ as follows: $\Phi_{i}=\phi_{\beta(i)}$, and $\gamma(i) \equiv \beta \delta(0)-i(i \in I)$. Then we have

$$
\delta=\beta \cdot \gamma .
$$

From (i) and (iii) one gets equations

$$
\eta_{\delta(i)}=P_{2 i}+P_{2 i+1} \text { and } \eta_{i+1}=P_{2 i+1}+P_{2 i+2} \text {, }
$$

which show that the edges $\eta_{\delta(i)}$ and $\eta_{i+1}$ join successive pairs of vertices in $P_{2 i}$, $P_{2 i+1}, P_{2 i+2}$. Thus the sequence

$$
P_{0}, P_{1}, P_{2}, \cdots P_{2 e-1}, P_{0}
$$

describes a circular "walk" around $\Gamma$, accomplished in $2 e$ "steps" $P_{n} \rightarrow P_{n+1}$ $(n=0, \cdots, 2 e-1)$, so that the $e$ edges of $\Gamma$ are each traversed exactly twice, in the order

$$
\eta_{\delta(u)}, \eta_{1}, \eta_{\delta(1)}, \cdots, \eta_{e-1}, \eta_{\delta(e-1)}, \eta_{0}
$$

It is clear that every vertex must be reached at least once during the walk.

The permutation $\delta$ is an interesting invariant of B, since it determines $\Gamma$ as abstract tree. Indeed $\Gamma$ must be obtained from the cyclic graph of $2 e$ edges, oriented and labelled as shown, by identifying each pair of edges which carry the same label in such a way that the two orientations "cancel each other out". 


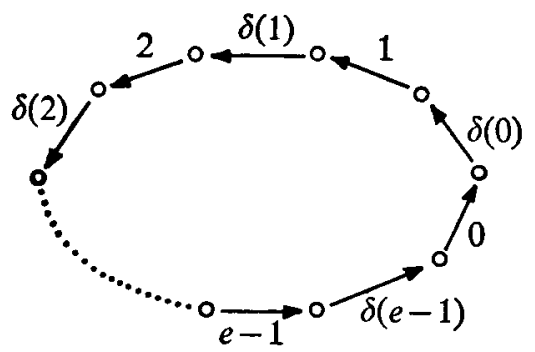

Of course this procedure can be carried out for any permutation $\delta$ of the set $I$, but will not necessarily give a tree.

We leave the reader to prove the following, as an application of the formula (1.2b) (see also [2, theorem 14]).

Corollary to theorem 2. Let B be a self-dual block. Then $\Gamma$ is an "open polygon" (i.e. an unbranched chain) if and only if either (1) every $\phi_{i}$ is real, so that $\beta(i)=i$ for all $i \in I$, or (2) $e$ is even, and $\beta(i) \equiv \frac{1}{2} e+i$ for all $i \in I$.

Recently Alperin and Janusz ([1]) have obtained results for the case $\mathbf{B}=\mathbf{B}_{0}(G)$ which are closely related to those in theorem 2 . They show that $A_{0}=R_{G}$ has a projective $R G$-resolution which is periodic of period $2 e$ :

$$
\cdots \rightarrow U\left(E_{2 e-1}\right) \rightarrow \cdots \rightarrow U\left(E_{1}\right) \rightarrow U\left(E_{0}\right) \rightarrow A_{0} \rightarrow 0 .
$$

Each $U\left(E_{n}\right)$ is indecomposable, with character $E_{n}$, and Alperin and Janusz have observed that

$$
E_{0}, E_{1}, E_{2} \cdots, E_{2 e-1}
$$

are the steps in a "walk" around $\Gamma$, during which each edge of $\Gamma$ appears exactly twice; they also give a rule for defining the sequence (1.3a), which is based on Janusz's classification of the indecomposable $k G$-modules in B. In fact the sequences (1.2d) and (1.3a) must coincide since by Schanuel's lemma ([10, p. 167]) the terms of any minimal projective resolution of $A_{0}$ are uniquely determined up to $R G$-isomorphism.

Theorem 2 is proved in section 7 , and the proof is based on theorem 1 , which is stated and proved in section 6. Theorem 1 gives information on certain indecomposable $k G$-modules, and explains how the permutation $\delta$ arises. The proof of theorem 1 does not use Janusz's classification, but is based on Dade's description of the indecomposable $k H$-modules, where $H=N_{G}\left(D_{d-1}\right)$ and $D_{d-1}$ is the subgroup of order $p$ of $D$ ([3, section 5]). This information about $k H$ modules is summarised in section 5 ; in case $d=1$, of course, it can be obtained more directly than by Dade's general argument. The passage from $H$ to $G$, which is made in the proof of theorem 1, uses methods originating with Thompson [12], 
and developed in [6] by Feit. Sections $2,3,4$, contain a summary of this general theory, as far as it is needed in this paper.

\section{S-projective maps and modules}

Throughout this section, $G$ is an arbitrary finite group, and $k$ an arbitrary commutative ring with 1 . Modules, both here and throughout the paper, are right and finitely-generated.

Let $\mathcal{S}=\left\{S_{\mu} \mid \mu \in M\right\}$ be a set of subgroups of $G$. We recall that a $k G$-module $U$ is said to be $\Xi$-projective if there exists for each $\mu \in M$ a $k S_{\mu}$-module $A_{\mu}$, so that $U$ is isomorphic to a direct summand of $\bigoplus_{\mu \in M} A_{\mu}^{G} . U$ is $\mathfrak{S}$-projective-free if it has no non-zero $\widetilde{S}$-projective direct summand. (If $\mathcal{S}$ consists of a single subgroup $S$, we write $S$-projective, $S$-projective-free rather than $\mathfrak{S}$-projective, $\subseteq$-projectivefree, and make a similar convention for all the notation of this section.)

If $U, V$ are $k G$-modules let $(U, V)$ be the $k$-module of all $k$-maps from $U$ to $V$. If $\theta \in(U, V)$ and $g \in G$, let $\theta^{g} \in(U, V)$ be the map $u \leftrightarrow\left(\left(u g^{-1}\right) \theta\right) g(u \in U)$. For any subgroup $S$ of $G$, write

$$
(U, V)_{s}=\left\{\theta \in(U, V) \mid \theta^{s}=\theta, \text { all } s \in S\right\} .
$$

Thus $(U, V)_{S}$ is the set of all $k S$-maps from $U$ to $V$.

The relative norm map $T_{S . G}:(U, V)_{S} \rightarrow(U, V)_{G}$ is the $k$-map defined by $T_{S, G}(\sigma)=\sum \sigma^{g}$, for $\sigma \in(U, V)_{S}$, the sum being over a transversal of the cosetspace $G / S=\{S g \mid g \in G\}$.

Definition. A $k G$-map $\theta: U \rightarrow V$ is $\subseteq$-projective if it belongs to

$$
(U, V)_{G G}=\sum_{\mu \in M} T_{S}{ }_{G}\left((U, V)_{S . .}\right)
$$

The $\Xi$-projective $k G$-maps form an "ideal" in the category $\mathscr{M}(k G)$ of all $k G$-modules and all $k G$-maps, i.e.

(2.1) Let $U, V, W$ be $k G$-modules, and let $\theta \in(U, V)_{G}, \phi \in(V, W)_{G}$. Then $\theta \phi$ is $\mathfrak{S}$-projective, if either $\theta$ or $\phi$ is $\mathfrak{S}$-projective.

In particular $(U, U)_{S . G}$ is an ideal, in the usual sense, of the $k$-algebra $(U, U)_{G}$. For proofs of these facts, which are nearly trivial, see [7, section 1$]$.

The following theorem of Higman and Dress gives the connection between $\mathfrak{S}$-projectivity of maps and $\mathfrak{S}$-projectivity of modules.

(2.2) (See $[4$, lemma 51.2] and [5, theorem 1]) $A k G$-module $U$ is S-projective, if and only if the identity map $\iota_{U}$ on $U$ is an $\mathcal{S}$-projective $k G$-map. This is equivalent to $(U, U)_{G}=(U, U)_{G . G}$. 
Definition. For any $k G$-modules $U, V$ let $(U, V)_{G}^{\subseteq}$ denote the $k$-module $(U, V)_{G} /(U, V)_{G, G}$.

We can now rewrite (2.2) as

(2.3) $A k G$-module $U$ is $\mathfrak{S}$-projective if and only if $(U, U)_{G}^{\mathfrak{S}}=0$.

\section{Some homological lemmas}

These are some elementary pieces of homological algebra which are useful in calculating $(U, V)_{G}^{\subseteq}$ in the very special case $\subseteq=\{1\}$, where 1 is the unit subgroup of $G$. In this case we use the term projective (instead of 1-projective). We shall also assume $k$ is a field. Throughout this section $U, V$ denote arbitrary $k G$-modules.

(3.1) Let $\pi: Q \rightarrow V$ be a projective presentation of $V$, i.e. $Q$ is a projective $k G$-module and $\pi$ is a surjective $k G$-map. Let $\theta \in(U, V)_{G}$. Then $\theta$ is projective if and only if there exists $\phi \in(U, Q)_{G}$ such that $\theta=\phi \pi$.

Proof. If such a $\phi$ exists, then $\theta=\phi \pi=\phi \cdot \iota_{Q} \cdot \pi$. But $\iota_{Q}$ is is projective by (2.2), so $\theta$ is projective by (2.1). Conversely, suppose that $\theta$ is projective. Then $\theta=T_{1, G}(\alpha)$ for some $\alpha \in(U, V)$. Since $V$ is a free $k$-module, there exists $\beta \in(V, Q)$ such that $\beta \pi=\iota_{V}$. Then by a trivial calculation $\theta=\phi \pi$, where $\phi=T_{1, G}(\alpha \beta)$.

The category $\mathscr{M}(k G)$ is self-dual, by means of the contragredient functor which takes $U$ to the usual dual $k G$-module $U^{*}=(U, k)$. Since $U^{*}$ is free, if and only if $U$ is free, the classes of projective and injective $k G$-modules coincide. Thus (3.1) automatically gives a dual version

(3.1*) Let $\pi^{\prime}: U \rightarrow Q^{\prime}$ be an injective embedding of $U$, i.e. $Q^{\prime}$ is an injective (= projective) $k G$-module and $\pi^{\prime}$ is an injective $k G-m a p$. Let $\theta \in(U, V)_{G}$. Then $\theta$ is projective if and only if there exists $\phi \in\left(Q^{\prime}, V\right)_{G}$ such that $\theta=\pi^{\prime} \phi$.

Denote by $\Phi(U)$ the Frattini submodule of $U$, i.e. the intersection of all maximal submodules of $U$; denote by $\Sigma(U)$ the socle of $U$, the sum of all minimal submodules of $U$. We recall that a projective presentation $\pi: Q \rightarrow U$ is minimal if $Q$ is minimal (among all such presentations of $U$ ); such minimal presentations always exist, and have the property $\operatorname{Ker} \pi \leqq \Phi(Q)$.

(3.2) Suppose that $U$ is projective-free, and that $\theta: U \rightarrow V$ is a surjective $k G$-map. Then $\theta$ is not projective unless $\theta=0$.

Proof. Let $\pi: Q \rightarrow V$ be a minimal projective presentation of $V$. If $\theta$ is projective, then by (3.1) there exists $\phi \in(U, Q)_{\mathrm{G}}$ such that $\theta=\phi \pi$. Thus $(\operatorname{Im} \phi) \pi$ $=\operatorname{Im} \theta=V$, hence $\operatorname{Im} \phi+\operatorname{Ker} \pi=Q$. But $\operatorname{Ker} \pi \leqq \Phi(Q)$, hence $\operatorname{Im} \phi+\Phi(Q)$ $=Q$, so by a standard property of Frattini modules, $\operatorname{Im} \phi=Q$. This implies that 
$U$ has a direct summand isomorphic to $Q$ (see [4, lemma 45.2]), and since $U$ is projective-free, we must have $Q=0$. Therefore $V=0$, and $\theta=0$.

The dual version is

(3.2*) Suppose that $V$ is projective-free, and that $\theta: U \rightarrow V$ is an injective $k G-m a p$. Then $\theta$ is not projective unless $\theta=0$.

(3.3) $(U, V)_{1, G}=0$ in each of the two cases

(1) $U$ projective-free and $V$ simple, or

(2) $U$ simple and $V$ projective-free.

Proof. Suppose if possible that $\theta \in(U, V)_{1 . G}$ and $\theta \neq 0$. In case (1), $\theta$ must be surjective since $V$ is simple; this contradicts (3.2). Similarly, case (2) leads to a contradiction of $\left(3.2^{*}\right)$.

(3.3) has as immediate corollary algebras.

(3.4) If $U$ is simple and non-projective, then $(U, U)_{G}^{1} \cong(U, U)_{G}$ as $k$ -

We conclude this section with a note on Hellers's $\Omega$-functor (Heller, [8]). If $U$ is a $k G$-module, define $\Omega U$ to be the kernel of a minimal projective presentation $\pi: Q \rightarrow U$, so that there is a $k G$-short exact sequence

$$
0 \rightarrow \Omega U \rightarrow Q \stackrel{\pi}{\rightarrow} U \rightarrow 0 .
$$

By Schanuel's lemma ([10, p. 167]) $\Omega U$ is defined up to isomorphism by (3.5a), and the fact that $\pi$ is minimal. We can make exactly the same construction for $R G$-lattices, where $R$ is the ring defined in section 1 . Heller has proved

(3.5) Let $U$ be a $k G$-module, or a $R G$-lattice. Then if $U$ is indecomposable and non-projective, so is $\Omega U$.

We say that a $k G$-module $U$ can be "lifted" to an $R G$-lattice $M$, if $\bar{M} \cong U$, where $\bar{M}=M / \mathfrak{p} M$ and $\mathfrak{p}$ is the maximal ideal of $R$. Every projective $k G$-module $Q$, can be lifted to a projective $R G$-lattice $P$.

(3.6) Suppose there is a $k G$-short exact sequence

$$
0 \rightarrow V \rightarrow Q \rightarrow U \rightarrow 0
$$

with $Q$ projective, and that $Q, U$ can be lifted to $P, M$ as above. Then $V$ can be lifted to an RG-lattice $N$, and there is an $R G$-short exact sequence

$$
0 \rightarrow N \rightarrow P \rightarrow M \rightarrow 0,
$$

which, in an obvious sense, "lifts" (3.6b).

Proof. This follows easily from the projective property of $P$. 


\section{The functors $f$ and $g$}

In this section $D$ is any $p$-subgroup of $G$, and $H$ any subgroup of $G$ which contains $N_{G}(D)$. Define

$$
\mathfrak{X}=\left\{D \cap D^{g} \mid g \in G \backslash H\right\}, \quad \mathfrak{V}=\left\{H \cap D^{g} \mid g \in G \backslash H\right\},
$$

so that these are sets of subgroups of $H$. We state the results below for the case where $k$ is a field of characteristic $p$; they hold also with $k$ replaced by the ring $R$ of section 1. Proofs of (4.1) to (4.4) are in [7].

(4.1) (i) Let $U$ be a D-projective $k G$-module. Then there exists a $\mathfrak{V}$-projective-free $k H$-module $f U$, and a $\mathfrak{Y}$-projective $k H$-module $U_{0}$, such that

$$
U_{H} \cong f U \oplus U_{0} .
$$

(ii) Let $L$ be a $D$-projective $k H$-module. Then there exists an $\mathfrak{X}$-projectivefree $k G$-module $g L$, and an $\mathfrak{X}$-projective $k G$-module $L_{0}$, such that

$$
L^{G} \cong g L \oplus L_{0} .
$$

Notice that $f U, g L$ are determined up to isomorphism, by the Krull-Schmidt theorem.

Now define $\mathfrak{A}$ to be the set of all subgroups $S$ of $D$, which are not $G$-conjugate to a subgroup of any $X$ in $\mathfrak{X}$.

(4.2) Suppose that $U, L$ above are both indecomposable, and have vertices $D_{0}, D_{1} \in \mathfrak{A}$. Then $f U, g L$ are both indecomposable, and have vertices $D_{0}, D_{1}$ respectively. Moreover

$$
\begin{aligned}
& g(f U) \cong U, \text { and } \\
& f(g L) \cong L .
\end{aligned}
$$

This shows that $f, g$ determine a one-one, vertex-preserving correspondence between the $k G$-isomorphism classes of indecomposable $k G$-modules with vertex in $\mathfrak{A}$, and the $k H$-isomorphism classes of indecomposable $k H$-modules with vertex in $\mathfrak{A}$. This module correspondence determined by $(G, H, D)$ is closely connected with Brauer's famous block correspondence (see [4, theorem 58.3]) between the $k G$-blocks of defect group $D$, and the $k H$-blocks of defect group $D$.

(4.3) Let $U, L$ be as in (4.2). Let B, $\mathbf{B}^{\prime}$ be, respectively, $k G, k H$-blocks of defect group $D$, which correspond under Brauer's block correspondence. Then

(4.3a) $U$ belongs to $\mathbf{B}$ if and only iff $U$ belongs to $\mathbf{B}^{\prime}$, and

(4.3b) L belongs to B' if and only if $g L$ belongs to B. 
(4.3) is proved in [7, theorem 5.8]. (It is clear from (4.2) that each of (4.3a), (4.3b) implies the other.)

Finally we may apply $f$ and $g$ to maps, in a functorial way. For each $k G$ module $U$, choose a decomposition (4.1a), with projection $p_{U}: U_{H} \rightarrow f U$ and injection $i_{U}: f U \rightarrow U_{H}$. If $U, V$ are $k G$-modules and $\theta: U \rightarrow V$ is a $k G$-map, we define the $k H$-map $f \theta: f U \rightarrow f V$ by

$$
f \theta=i_{U} \cdot \theta_{H} \cdot p_{V} .
$$

Here $\theta_{H}$ is $\theta$, regarded as $k H$-map.

(4.4) Let $U, V$ be D-projective $k G$-modules, and let $\theta: U \rightarrow V$ be an arbitrary $k G-m a p$. Then

(i) $f \iota_{U}=\iota_{f U}$.

(ii) $\theta \mapsto f \theta$ induces a $k$-isomorphism

$$
(U, V)_{G}^{\mp} \cong(f U, f V)_{H}^{\boldsymbol{x}} .
$$

In case $U=V$, this is an isomorphism of k-algebras.

One can also define $g \alpha: g L \rightarrow g M$, for any $k H$-map $\alpha: L \rightarrow M(L, M k H$ modules), and prove an analogue to (4.4). However we do not need this, and so we do not give it.

Our final lemma (4.5) shows that $f, g$ "commute" with the functor $\Omega$. We leave the proof to the reader. (In fact we shall need only the formula (4.5b), and only in a case where $\mathfrak{X}=\{1\}$. This can be proved by an application of Schanuel's lemma.) Then

(4.5) Let $U$ be a $D$-projective $k G$-module, and $L$ a $D$-projective $k H$-module.

$$
\begin{aligned}
& f \Omega U \cong \Omega f U, \text { and } \\
& g \Omega L \cong \Omega g L .
\end{aligned}
$$

\section{Indecomposable $\boldsymbol{k H}$-modules in $\mathrm{B}^{\prime}$}

From now on, $G, \mathrm{~B}, D, k, R$ are as in section 1 . Let $H=N_{G}\left(D_{d-1}\right)$, where $D_{d-1}$ is the subgroup of $D$ of order $p$. Since $H \geqq N_{\mathrm{G}}(D)$, the correspondences of section 4 apply.

Let B' be the $k H$-block corresponding to B. In this section we give Dade's results on indecomposable $k H$-modules in $\mathbf{B}^{\prime}$.

Let $C=C_{G}\left(D_{d-1}\right)^{(1)}$. Dade shows $([3$, section 1$])$ that there is a $k C$-block b

(1) The table below gives equivalents in [3], for notations used in section 5 .

$$
\begin{array}{rlllllll}
\text { Section 5: } d & q & D_{d-1} & H & C & F & \mathbf{B}^{\prime} & \mathbf{b} \\
\text { [3]: } a & p^{a} & D_{a-1} & N_{a-1} & C_{a-1} & E . C_{a-1} & B_{a-1} & b_{a-1}
\end{array}
$$


such that $\mathbf{b}^{H}=\mathbf{B}^{\prime}$, and that all such $k C$-blocks are conjugate in $H$. The stabilizer $F$ of $\mathbf{b}$ in $H$ has the form $F=E \cdot C$, where $E$ is a certain subgroup of $N_{G}(D) ; F / C$ is cyclic of order $e$ dividing $p-1$, because it is a subgroup of $H / C$ which is isomorphic to a subgroup of $\operatorname{Aut}\left(D_{d-1}\right)$. We may use this as our definition of $e$, or alternatively use Dade's definition in [3, section 1$]$, and use [3, lemma 1.4].

Write $q=p^{d}$, and $I=\{0,1, \cdots, e-1\}$.

(5.1) (i) B' contains e simple $k H$-modules $S_{i}(i \in I)$, such that every simple $k H$-module in $\mathbf{B}^{\prime}$ is isomorphic to exactly one $S_{i}$. Let $T_{i}(i \in I)$ be projective indecomposable $k H$-modules, numbered so that $T_{i} / \Phi\left(T_{i}\right) \cong S_{i}(i \in I)$.

(ii) There is a multiplicative isomorphism $\sigma \mapsto \bar{\sigma}(\sigma \in D)$ of $D$ into the centre of $k C$, such that if $\alpha$ is a generator of $D$ then for any $i \in I$

$$
T_{i}>T_{i}(\bar{\alpha}-1)>T_{i}(\bar{\alpha}-1)^{2}>\cdots>T_{i}(\bar{\alpha}-1)^{q-1}>T_{i}(\bar{\alpha}-1)^{q}=0
$$

is the unique $k H$-composition series of $T_{i}$.

(iii) Every indecomposable $k H$-module in $\mathbf{B}^{\prime}$ is isomorphic to exactly one of the following

$$
T_{i, v}=T_{i} / T_{i}(\bar{\alpha}-1)^{\nu}(i \in I, v \in\{1, \cdots, q\}) .
$$

In particular $S_{i} \cong T_{i, 1}$ and $T_{i} \cong T_{i, q}$, for all $i \in I$.

Proof. For each $\sigma \in D$, take $\bar{\sigma}$ to be the residue class mod $\mathfrak{p} \cdot D C$ of the element $\tilde{\sigma}$ defined in $[3,(5.3)]$. We may interpret $\bar{\sigma}$ as an element in $k C$. All parts of (5.1) now follow from [3, section 5].

(5.2) Let $S, S^{\prime}$ be simple $k H$-modules in $\mathbf{B}^{\prime}$, such that $S_{F} \cong S_{F}^{\prime}$. Then $S \cong S^{\prime}$.

Proof. Any simple component of $S_{C}$ or $S_{C}^{\prime}$ is the unique (up to isomorphism) simple $k C$-module in some $H$-conjugate of $\mathbf{b}$, and hence has $F$ as its stabilizer in $H$. Now (5.2) follows from Clifford's theorem (see [4, theorem 14.1]).

We need information on the order of the composition factors in (5.1a). Let $\alpha$ be a generator of $D$, and let $\alpha_{1}=\alpha^{p d-1}$. Since $H$ normalises $D_{d-1}=\left\langle\alpha_{1}\right\rangle$, there is a linear representation $\psi: H \rightarrow k^{*}$, given by

$$
\psi(h)=n(h) \cdot 1_{k}(h \in H),
$$

where $n(h) \in Z$ is defined up to congruence $\bmod p$ by

$$
\alpha_{1}^{h}=\alpha_{1}^{n(h)} \quad(h \in H) .
$$

Evidently $\psi(c)=1$, for all $c \in C$, and hence $\psi^{[H: C]}=1$, the trivial representation of $H$.

Now let $z$ be an element of $E$. Since $z$ normalises $D$, 


$$
\alpha^{z}=\alpha^{n(z)},
$$

where $n(z) \in Z$ is defined up to congruence $\bmod p^{d}$. Since (5.3c) implies $\alpha_{1}^{z}=\alpha_{1}^{n(z)}$, there is no conflict between (5.3b) and (5.3c). From $[3,(5.3)]$ we find that $(\bar{\sigma})^{z}=\overline{\sigma^{z}}$, for all $\sigma \in D$. Taking $\sigma=\alpha$ and using (5.3c) we have

$$
\bar{\alpha}^{z}=\bar{\alpha}^{n(z)} \quad(z \in Z) .
$$

In the next theorem, $\psi^{v}(v \in Z)$ denotes, abusively, a 1-dimensional $k H$-module which affords the representation $\psi^{v} ; \otimes=\otimes_{k}$ is the usual "external tensor product" of $k H$-modules. In particular, $\psi^{v} \cong \psi \otimes \cdots \otimes \psi(v$ factors $\psi)$, if $v>0$.

(5.4) (i) Let $S_{i, v}=T_{i}(\bar{\alpha}-1)^{v} / T_{i}(\bar{\alpha}-1)^{v+1}$, for given $i \in I$ and $v \in\{0 \cdots, q-1\}$. Then $S_{i, v} \cong \psi^{v} \otimes S_{i}$.

(ii) Write $S_{n}=\psi^{n} \otimes S_{0}$, for all $n \in Z$. Then $S_{m} \cong S_{n}$ if and only if $m \equiv n \bmod e$, and we can take $S_{0}, \cdots, S_{e-1}$ for the set of simple $k H$-modules in $\mathbf{B}^{\prime}$ mentioned in (5.1) (i).

(iii) With the notation just given, the composition factors of $T_{i}$ (see (5.1a)) are $S_{i}, S_{i+1}, \cdots, S_{i+q-1} \cong S_{i}$.

Proof. (i) Let $t \in T_{i}$ and $z \in E$. Then using (5.3a) - (5.3d) one finds

$$
\begin{aligned}
t(\bar{\alpha}-1)^{v} z & =t z\left(\bar{\alpha}^{n(z)}-1\right)^{v}=t z(\bar{\alpha}-1)^{v}\left(1+\bar{\alpha}+\cdots+\bar{\alpha}^{n(z)-1}\right)^{v} \\
& \equiv t z(\bar{\alpha}-1)^{v} \cdot n(z)^{v} \bmod T_{i}(\bar{\alpha}-1)^{v+1}
\end{aligned}
$$

and hence

$$
t(\bar{\alpha}-1)^{v} z \equiv \psi^{\nu}(z) \cdot t z(\bar{\alpha}-1)^{v} \bmod T_{i}(\bar{\alpha}-1)^{v+1},
$$

for all $z \in E$. But (5.4a) holds also for all $z \in C$, since then $z$ commutes with $\bar{\alpha}$, and $\psi(z)=1$. Hence (5.4a) holds for all $z$ in $F=E \cdot C$ and it is easy to deduce that $\left(S_{i, v}\right)_{F} \cong\left(\psi^{v} \otimes S_{i}\right)_{F}$. Therefore $S_{i, v} \cong \psi^{v} \otimes S_{i}$ by $(5.2)$.

(ii) Since $\psi^{[H: C]}=1$, we have $S_{m} \cong S_{n}$ if $m \equiv n \bmod [H: C]$. Now $[H: C]$ divides $p-1$, therefore is less than $q$, and so it follows from (i) that all the modules

$$
S_{0}, S_{1}, S_{2}, \cdots
$$

are composition factors of $T_{0}$, and hence lie in $\mathbf{B}^{\prime}$. Conversely, let $S$ be any simple $k H$-module in $\mathbf{B}^{\prime}$. By Brauer's theorem [4, theorem 46.2] there exists a finite sequence of elements $i_{0}, i_{1}, \cdots, i_{r} \in I$, such that $S_{i_{n}}=S_{0}, S_{i_{r}} \cong S$, and for each $\rho \in\{1, \cdots, r\}, S_{i \rho}$ is a composition factor of $T_{i \rho-1}$. Then (i) shows that for each $\rho, S_{i \rho} \cong \psi^{v(\rho)} \otimes S_{i \rho-1}$ for some $v(\rho) \in Z$. Therefore $S \cong \psi^{n} \otimes S_{0}$ for some $n \in Z$, and hence $S$ is isomorphic to some module in (5.4b). We now know (by (5.1)(i)) that (5.4b) contains exactly $e$ non-isomorphic modules, and all the statements of (ii) follow. 
(iii) is an immediate consequence of (i), (ii) and (5.1a).

(5.4) (ii) allows us to adopt, as we shall do henceforth, the following

Notation. $S_{i}$ is defined for all $i \in Z$, in such a way that $S_{i} \cong \psi^{i} \otimes S_{0}$; hence $S_{i+e} \cong S_{i}(i \in Z)$. Similarly $T_{i}, T_{i, v}$ are defined for all $i \in Z$, in such a way that (5.1) holds, and $T_{i+e} \cong T_{i}, T_{i+e . v} \cong T_{i, v}(i \in Z, v \in\{1, \cdots q\})$.

(5.5) Let $i \in I, v \in\{1, \cdots, q\}$. Then

(i) $T_{i, v}$ is projective if and only if $v=q$.

(ii) There is a $k H$-short exact sequence

$$
0 \rightarrow T_{i+v, q-v} \rightarrow T_{i, q} \rightarrow T_{i, v} \rightarrow 0 .
$$

(iii) If $1 \leqq v \leqq q-1, \Omega T_{i, v} \cong T_{i+v . q-v}$.

(iv) $\Omega^{2} S_{i} \cong S_{i+1}$.

Proor. (i) and (ii) follow from (5.1), (5.4). (iii) follows from the definition of the $\Omega$-functor, and the fact that $T_{i, q} \rightarrow T_{i, v}$ is a minimal projective presentation if $1 \leqq v \leqq q-1$. Then (iv) follows from (iii) and the fact that $S_{i} \cong T_{i 1}$, all $i$.

We conclude with a remark on the dimensions of the modules $S_{i}$. Let $\operatorname{dim} S_{0}=N_{0}$. Then $\operatorname{dim} S_{i}=N_{0}$ for all $i$, since $S_{i} \cong \psi^{l} \otimes S_{0}$. Let $\pi(n)$, for any positive integer $n$, denote the exponent of the highest power of $p$ which divides $n$. A general theorem of Brauer on blocks ([4, theorem $61.6(2)])$ now gives at once

$$
\pi\left(N_{0}\right)=\pi(|H|)-d
$$

\section{The permutation $\delta$}

Take $G, D, H, \mathbf{B}, \mathbf{B}^{\prime}$ as in section 5 . We shall apply the module correspondence of section 4 , using the notation $f, g, \mathfrak{X}, \mathfrak{Y}, \mathfrak{A}$ there. In our case $\mathfrak{X}=\{1\}$, since it is clear that $D \cap D^{g}=1$ for any $g \in G \backslash H$. In general $\mathfrak{Y} \neq\{1\}$. The set $\mathfrak{A}$ consists of all the subgroups of $D$ except 1 .

THEOREM 1. (i) B contains e simple kG-modules $V_{i}(i \in I)$, such that every simple $k G$-module in $\mathrm{B}$ is isomorphic to exactly one $V_{i}$. Let $W_{i}(i \in I)$ be projective indecomposable $k G$-modules such that $W_{i} / \Phi\left(W_{i}\right) \cong V_{i}(i \in I)$.

(ii) The numbering of the $V_{i}(i \in I)$ can be arranged so that

(6.1a) $\quad\left(f V_{j}, S_{i}\right)_{H} \cong\left(V_{j}, g S_{i}\right)_{G} \cong k$ or zero, according as $i=j$ or $i \neq j$, and there is a permutation $\delta$ of $I$ such that

(6.1b) $\quad\left(S_{i}, f V_{j}\right)_{H} \cong\left(g S_{i}, V_{j}\right)_{G} \cong k$ or zero, according as $\delta(i)=j$ or $\delta(i) \neq j$, for all $i, j \in I$.

(iii) For each $i \in I$ there exist $k G$-short exact sequences 


$$
\begin{aligned}
& \mathbf{F}_{2 i}: \quad 0 \rightarrow \Omega g S_{i} \rightarrow W_{\delta(i)} \rightarrow g S_{i} \rightarrow 0 . \\
& \mathbf{F}_{2 i+1}: 0 \rightarrow g S_{i+1} \rightarrow \bar{W}_{i+1} \rightarrow \Omega g S_{i} \rightarrow 0,
\end{aligned}
$$

Theorem 1 is proved below, in a series of lemmas. Let $V_{j}(j \in J)$ be a full set of mutually non-isomorphic simple $k G$-modules in $B$, indexed by a suitable finite set $J$. All the $S_{i}$ and $V_{j}$ are $D$-projective, since they belong to blocks with $D$ as defect group ([4, theorem 54.10]). On the other hand no $S_{i}, V_{j}$ is projective, since a simple, projective $k G$-module $S$ (for any finite group $G$ ), must lie in a block of defect group $1^{(2)}$. Therefore each $S_{i}, V_{j}$ has vertex in $\mathfrak{A}$, and we can apply (4.2), (4.3) to prove

(6.2) $f V_{j}$ is indecomposable, non-projective and lies in $\mathbf{B}^{\prime} . g S_{i}$ is indecomposable, non-projective and lies in B.

We have now, for any $i \in I, j \in J$, that $\left(S_{i}, f V_{j}\right)_{H}^{1} \cong\left(g S_{i}, V_{j}\right)_{G}^{1}$ by (4.2), (4.4a). But (3.3) gives also $\left(S_{i}, f V_{j}\right)_{H}^{1} \cong\left(S_{i}, f V_{j}\right)_{H}$, and $\left(g S_{i}, V_{j}\right)_{G}^{1} \cong\left(g S_{i}, V_{j}\right)_{G}$. This proves the first part of (6.3) below, and the second part is proved similurly.

(6.3) $\left(S_{i}, f V_{j}\right)_{H} \cong\left(g S_{i}, V_{j}\right)_{G}$ and $\left(f V_{j}, S_{i}\right)_{H} \cong\left(V_{j}, g S_{i}\right)_{G}$.

(6.4) There is a map $h: J \rightarrow I$ such that for all $i \in I, j \in J$,

(6.4a) $h(j)=i$ if and only if $\left(f V_{j}, S_{i}\right)_{H} \neq 0$. Moreover $h$ is a bijection, and hence $|J|=|I|=e$.

Proof. Take any $j \in J$. By (6.2), (5.1), (5.5) we have $f V_{j} \cong T_{h(j) . v(j)}$ for some $h(j) \in I$ and some $v(j) \in\{1, \cdots, q-1\}$. Since $T_{h(j) v(j)}$ is uniserial, with "top" composition factor $S_{h(j)}$, one has by Schur's lemma

(6.4b) $\left(f V_{j}, S_{i}\right)_{H} \cong k$ or zero, according as $h(j)=i$ or $h(j) \neq i$.

This establishes the existence of $h$, and proves (6.4a).

Now suppose $i \in I$ is given. Take any minimal submodule $S$ of $g S_{i}$. Since $S$ is in B, there exists $j \in J$ such that $V_{j} \cong S$. This implies $\left(V_{j}, g S_{i}\right)_{G} \neq 0$, hence $\left(f V_{j}, S_{i}\right)_{H} \neq 0$ by (6.3). Thus $h(j)=i$. We have now proved that $h$ is surjective.

Suppose $j, j^{\prime} \in J$ are such that $h(j)=i=h(j)$. Then $f V_{j}=T_{i, v}$ and $f V_{j^{\prime}}=T_{i, 0^{\prime}}$ for some $v, v^{\prime} \in\{1, \cdots, q-1\}$. We may assume $v \geqq v^{\prime}$. But then there exists a surjective $k H$-map $\theta: T_{i, v} \rightarrow T_{i, v^{\prime}}$, and by (3.2) $\theta$ is not projective. Therefore $\left(f V_{j}, f V_{j^{\prime}}\right)_{H}^{1} \neq 0$. By $(4.4 a)\left(V_{j}, V_{j}\right)_{G}^{1} \neq 0$, hence $\left(V_{j}, V_{j^{\prime}}\right)_{G} \neq 0$. Schur's lemma now gives $j=j^{\prime}$. Therefore $h$ is a bijection.

We have now proved part (i) of theorem 1. From now on we take $J=I$ and

(2) Because $S$ is projective, its dimension is divisible by $p^{a}(a=\pi(|G|))$; also $S$ can be lifted to an $R G$-lattice $M$, for the same reason. The character $\chi$ of $M$ must be simple, because $S$ is simple. Since $p^{a}$ divides $\chi(1), \chi$, and hence also $S$, lies is a block of defect zero, i.e. of defect group 1 . 
arrange notation of the $V$ so that the map $h: I \rightarrow I$ is the identity. This means that we have for each $j \in I$

$$
f V_{j}=T_{j(j)} \text {, for some } v(j) \in\{1, \cdots, q-1\} .
$$

Formula (6.1a) in theorem 1 is now a consequence of (6.4b) and (6.3). But we may now prove a "dual" version of (6.4), which in the present notation will show that there is a map $\delta: I \rightarrow I$, such that for all $i, j \in I$, we have $\delta(i)=j$ if and only if $\left(S_{i}, f V_{j}\right)_{H} \neq 0$; moreover $\delta$ is a bijection, i.e. it is a permutation of $I$. The proof is exactly parallel to that of (6.4). In place of (6.4b) one has, using the uniseriality of $f V_{j}=T_{j \cdot(j)}$

$$
\left(S_{i}, f V_{j}\right)_{H} \cong k \text { or zero, according as } \delta(i)=j \text { or } \delta(i) \neq j .
$$

Now (6.4d) and (6.3) yield (6.1b), and we have proved part (ii) of theorem 1.

(6.1a), (6.1b) tell us that, for all $i \in I$,

$$
\Sigma\left(g S_{i}\right) \cong V_{i} \text { and } g S_{i} / \Phi\left(g S_{i}\right) \cong V_{\delta(i)} .
$$

From the second of these, and using the projective property of $\bar{W}_{\delta(i)}$, one can make a projective presentation $W_{\delta(i)} \rightarrow g S_{i}$. This must be minimal, since $W_{\partial(i)}$ is indecomposable. Therefore there exists a $k G$-short exact sequence $\mathbf{F}_{2 i}$.

From $\Sigma\left(g S_{i+1}\right) \cong W_{i+1},{ }^{(3)}$ and using the injective property of $\bar{W}_{i+1}$, one can make an injective embedding $g S_{i+1} \rightarrow W_{i+1}$. Hence there is some $k G$-module $V$, and a short exact sequence

$$
0 \rightarrow g S_{i+1} \rightarrow \bar{W}_{l+1} \rightarrow V \rightarrow 0 .
$$

On the other hand we deduce from (4.5b) and (5.5)(iv) that $\Omega\left(\Omega g S_{\imath}\right) \cong g\left(\Omega^{2} S_{i}\right)$ $\cong g S_{i+1}$. So there is a short exact sequence

$$
0 \rightarrow g S_{i+1} \rightarrow W \rightarrow \Omega g S_{i} \rightarrow 0,
$$

where $W$ is projective. The dual form of Schanuel's lemma now gives $V \cong \Omega g S_{i}$, and hence we can take $(6.4 e)$ as $F_{2 i+1}$. We have now proved all parts of theorem 1 .

REMARK. The integers $v(j)$ in (6.4c) satisfy the condition

$$
\text { For each } j \in I \text {, either } 1 \leqq v(j) \leqq e, \text { or } q-e \leqq v(j) \leqq q-1 \text {. }
$$

This result is due to Feit, and is froved by applying a lemma of Passman [6, lemma 4] to the condition $\left(T_{j v(j)}, T_{j, v(j)}\right)_{H}^{1} \cong k$, which in turn follows from Schur's lemma, and (4.4a) in the case $U=V=V_{j}$. Now $g T_{j} v(j) \cong V_{j}$, by (6.2), (4.2a), so that

(3) We extend the definition of $\bar{W}_{i}$ to any $i \in Z$, by the convention $\bar{W}_{i+e}=\bar{W}_{i}(i \in Z)$. See section 7. 


$$
T_{j . v(j)}{ }^{G} \cong V_{j} \oplus U_{0},
$$

where $U_{0}$ is a projective $k G$-module. By (5.1), (5.5), (5.6) we have $\operatorname{dim} T_{j . v(j)}{ }^{G}$ $=v(j) \cdot N$, where $N=N_{0} \cdot[G: H]$, and

$$
\pi(N)=a-d(a=\pi(|G|)) .
$$

On the other hand, $\operatorname{dim} U_{0} \equiv 0 \bmod p^{a}([4$, lemma 59.6]). So taking dimensions on both sides of $(6.5 \mathrm{~b})$, we have

$$
\operatorname{dim} V_{j} \equiv v(j) \cdot N \bmod p^{a} .
$$

Taking (6.5d) with (6.5a), we get a strengthened form of a theorem of Rothschild [11]. Feit points out in [6], that this theorem shows that all the $V_{j}$ have vertex $D$. However this is not necessary for the proof of theorem 2.

\section{Proof of theorem 2}

Define projective indecomposuble $R G$-lattices $W_{i}$, such the module $\bar{W}_{i}$ in theorem 1 lifts to $W_{i}$, for all $i \in I$. Then define $W_{i}$ for all $i \in Z$, by the rule $W_{i}$ $=W_{i+e}$ for all $i \in Z$. With the notations of section 5 , we can s $d y$ th the $k G$-short ex.ct sequences $\mathbf{F}_{2 i}, \mathbf{F}_{2 i+1}$ of theorem 1 exist for all $i \in Z$, and that $\mathbf{F}_{n} \cong \mathbf{F}_{n+2 e}$ $(n \in Z)$, with the usual isomorphism of short exact sequences. Write $B_{2 i}=g S_{i}$, $B_{2 i+1}=\Omega g S_{i}$, all $i \in Z$.

(7.1) Let $M$ be an $R G$-lattice and $m$ a fixed element of $Z$ such that $\bar{M} \cong B_{m}$. Then we can construct $R G$-lattices $A_{n}$ and sequences $\mathrm{E}_{n}$, with $A_{m}=M$, and $\bar{A}_{n} \cong B_{n}$ and $\mathbf{E}_{n}$ "lifts" $\mathbf{F}_{n}$, for all $n \in Z$.

Proof. By (3.6) we can lift $\mathbf{F}_{m}$ to an $R G$-short exact sequence $\mathbf{E}_{m}$ in which $A_{m}=M$, and $\bar{A}_{m+1} \cong B_{m+1}$. Now we c.n repeat the process with $m+1$ in place of $m$. So we define, inductively, $\mathbf{E}_{n}$ for all $n \geqq m$. Now we can apply (3.6) to the "dual" of $\mathbf{F}_{m-1}$, and regard the result as the dual of an $R G$ sequence $\mathbf{F}_{m-1}$, which lifts $F_{m-1}$. Proceeding in this way, we can define $\mathbf{E}_{n}$ for all $n<m$.

(7.2) Let $M, A_{n}$ be as in (7.1), and assume that the character $P_{m}$ of $M=A_{m}$ lies in $\Gamma_{v}$. Then the character $P_{n}$ of $A_{n}$ lies in $\Gamma_{v}$, for all $n \in Z$. Also $P_{n+2 e}=P_{n}$, for all $n \in \boldsymbol{Z}$.

Proof. We have equations (1.2c), for all $i \in Z$. Taking these together with equations (1.1a), it is clear that all the $P_{n}$ lie in $\Gamma_{v}$, as soon as $P_{m}$ does. To prove the final statement of (7.2), suppose first that $n=2 i(i \in Z)$. From (1.2c) we have

$$
\begin{gathered}
\eta_{\delta(i)}=P_{n} \quad+P_{n+1}, \\
\eta_{i+1}=P_{n+1}+P_{n+2}, \\
\cdots \quad \cdots
\end{gathered}
$$




$$
\begin{gathered}
\eta_{\delta(i+e-1)}=P_{-+2 e-2}+P_{n+2 e-1}, \\
\eta_{i+e}=P_{n+2 e-1}+P_{n+2 e} .
\end{gathered}
$$

Form the alternating sum of these $2 e$ equations. We find $0=P_{n}-P_{n+2 e}$, as required. A similar argument works in the case $n=2 i+1(i \in Z)$.

(7.3) Let $M, A_{n}$ be as in (7.2). Then $A_{n+2 e} \cong A_{n}$, for all $n \in Z$.

Proof. Let $i \in Z$, and let $K$ be the quotient field of $R$. From (1.1a) it is clear that $K \otimes_{R} W_{i}$ has unique $K G$-submodules, $Y_{i(1)}, Y_{i(2)}$ say, with characters $X_{i(1)}$, $X_{i(2)}$ respectively, and that these are the only $K G$-submodules of $K \otimes_{R} W_{i}$ which have characters in $\Gamma_{v}$. Thus $W_{i} \cap Y_{i(1)}, W_{l} \cap Y_{i(2)}$ are the only $R$-pure $R G$-submodules of $W_{i}$, which have characters in $\Gamma_{v}$. Fix $n \in Z$. Then $\mathbf{E}_{n-1}$ shows that $A_{n}$ is isomorphic to an $R$-pure $R G$-submodule of some $W_{i}$, similarly $\mathbf{E}_{n+2 e-1}$ shows that $A_{n+2 e}$ is isomorphic to an $R$-pure $R G$-submodule of $W_{i+e}=W_{i}$. But (7.2) shows that these submodules of $W_{i}$ have the same character. So they coincide, i.e. $A_{n+2 e} \cong A_{e}$.

(7.1), (7.2), (7.3) allow us to prove parts (i) and (iii) of theorem 1, as soon as we have an $R G$-lattice $M$ such that $\bar{M} \cong B_{m}$ for some $m$, and $M$ has character in $\Gamma_{v}$. If $\mathbf{B}=\mathbf{B}_{0}(G)$, the princip.l block of $G$, we just take $M=A_{0}=R_{G}$. In general we proceed is follows.

Let $M$ be the indecomposable $R G$-lattice in $\mathbf{B}$, which has character $X_{1}$, and is defined in [3, p. 39]. By [3, lemma 6.2] there is an indecomposable $R H$-lattice $L$ such that

$$
M_{H}=L \oplus Q_{1} \oplus \cdots \oplus Q_{1}{ }^{(4)}
$$

where the $Q_{j}$ are indecomposable $R H$-modules, each of which is either projective, or lies in a block other than $\mathbf{B}^{\prime}$. Both $\bar{M}, \bar{L}$ are shown to be indecomposable and it follows by taking (7.4) mod $p$, and using (4.1), (4.2), (4.3) that $\bar{L} \cong f \bar{M}$, and hence $\bar{M} \cong g \bar{L}$. But $\left(\left[3\right.\right.$, p. 39]) $L$ has character either $\tilde{X}_{i}(i \in I)$ or $\sum \widetilde{X}_{\lambda}$, where $\tilde{X}_{0}, \cdots, \tilde{X}_{e-1}, \tilde{X}_{\lambda}(\lambda \in \Lambda)$ are the ordinary characters of $\mathbf{B}^{\prime}$; now $[3$, theorem 4$]$ and (5.5)(iii) show that $\bar{L}$ is isomorphic either to $S_{i}$, or to $\Omega S_{i}$, for some $i \in I$. Therefore $\bar{M}$ is isomorphic either to $g S_{i}$ or to $\Omega g S_{i}$, i.e. to some $B_{m}$. Now (7.1), (7.2), (7.3) allow us to prove parts (i) and (iii) of theorem 1.

To prove part (ii) of theorem 2, observe that the construction used for (7.1) shows that $\bar{A}_{0}, \bar{A}_{1}, \cdots, \bar{A}_{2 e-2}, \bar{A}_{2 e-1}$ are isomorphic to

$$
g S_{0}, \Omega g S_{0}, \cdots, g S_{e-1}, \Omega g S_{e-1} \text {. }
$$

These modules are mutually non-isomorphic, provided $q=|D| \neq 2$, for in this case $S_{u}, \Omega S_{0}, \cdots, S_{e-1}, \Omega S_{e-1}$ are easily seen to be non-isomorphic. Hence $A_{0}$,

(4) $M, L, Q$, are denoted by corresponding script capitals in [3]. 
$\cdots, A_{2 e-1}$ are non-isomorphic, which proves (ii) in case $q \neq 2$. If $q=2$, then $A_{0} \cong A_{1}$, but an ad hoc argument proves that $A_{0} \not A_{1}$ anyway. For we have $p=2, e=1$ and $(q-1) / e=1$. Therefore equations (1.1a) reduce to the single equation $\eta_{0}=X_{1}+X_{2}$. But (7.2) gives $\eta_{0}\left(=\eta_{\delta(0)}\right)=P_{0}+P_{1}$. Hence $\left\{P_{0}, P_{1}\right\}$ $=\left\{X_{1}, X_{2}\right\}$, which shows that $P_{0} \neq P_{1}$, therefore $A_{0} \not A_{1}$. So (ii) holds in all cases.

It remains to prove part (iv) of theorem 2. We assume B is self-dual, and that $\beta$ is the permutation of $I$ given by $\bar{\phi}_{i}=\phi_{\beta(i)}$. Evidently $\beta=\beta^{-1}$. We have also

$$
\bar{W}_{i}^{*} \cong \bar{W}_{\beta(i)}
$$

for all $i \in I$, and by an obvious extension of $\beta$, we can say that (7.5) holds for all $i \in \boldsymbol{Z}$. Apply the dual functor to $\mathbf{F}_{2 i-1}$, and use (7.5). We get the $k H$-short exact sequence

$$
0 \rightarrow\left(\Omega g S_{i}\right)^{*} \rightarrow W_{\beta(i)} \rightarrow\left(g S_{i}\right)^{*} \rightarrow 0 .
$$

It is trivial that the functor $g$ commutes with $*$, hence $\mathbf{B}^{\prime}$ is self-dual, and so $S_{0}^{*} \cong S_{m}$ for some $m \in I$. Then by (5.5) one has for any $i \in I, S_{i}^{*} \cong\left(\psi^{i} \otimes S_{0}\right)^{*} \cong \psi^{i} \otimes$ $S_{m} \cong S_{m-i}$. Hence $\left(g S_{i}\right)^{*} \cong g S_{m-i}$. Now compare (7.6) with

$$
\mathbf{F}_{2(m-i)}: 0 \rightarrow g S_{m-i} \rightarrow \bar{W}_{\delta(m-i)} \rightarrow \Omega g S_{m-i} \rightarrow 0 .
$$

Sch nuel's lemma gives $\left.W_{\beta(i)} \cong W_{\delta(m-i}\right)$, and therefore $\beta(i) \equiv \delta(m-i) \bmod e$, for all $i \in Z$. Repl..ce $i$ by $m-i$; we have

$$
\delta(i) \equiv \beta(m-i) \bmod e, \text { all } i \in Z .
$$

If we put $i=0$ in (7.7), we find $\delta(0)=\beta(m)$, so $m=\beta^{-1} \delta(0)=\beta \delta(0)$. Thus (7.7) is the formula (1.2b) which we want.

\section{References}

[1] J. Alperin and H. Janusz, Resolutions and periodicity, Proc. Amer. Math. Soc. 37 (1973), 403-406.

[2] R. Brauer, 'Investigations on group characters', Ann. of Math. 42 (1941), 936-958.

[3] E. C. Dade, 'Blocks with cyclic defect groups', Ann. of Math. 84 (1966), $20-48$.

[4] L. Dornhoff, Group Representation Theory, Marcel Dekker, New York, 1972.

[5] A. Dress, 'Vertices of integral representations', Math. Z. 114 (1970), 159-169.

[6] W. Feit, Some properties of the Green correspondence, Theory of Finite Groups, Symposium, Harvard Univ., Cambridge, Mass., 1968.

[7] J. A. Green, 'Relative module categories for finite groups', J. Pure Applied Algebra 2 (1972), 371-393.

[8] A. Heller, 'The loop-space functor in homological algebra', Trans. Amer. Math. Soc. 96 (1960), 382-394.

[9] G. Janusz, 'Indecomposable modules for finite groups', Ann. of Math. 89 (1969), 209-241. 
[10] I. Kaplansky, Fields and Rings, University of Chicago Press, Chicago, Ill., 1969.

[11] B. Rothschild, 'Degrees of irreducible modular characters of blocks with cyclic defect groups', Bull. Amer. Math. Soc. 73 (1967), 102-104.

[12] J. Thompson, 'Vertices and sources', J. Algebra 6 (1967), 1-6.

\author{
Mathematics Institute \\ University of Warwick \\ Coventry CV4 7AL \\ England
}

